\title{
The Structure and Performance of an Open-Domain Question Answering System
}

\author{
Dan Moldovan, Sanda Harabagiu, \\ Marius Pasca, Rada Mihalcea, Roxana Girju, Richard Goodrum and Vasile Rus \\ Department of Computer Science and Engineering \\ Southern Methodist University \\ Dallas, Texas, 75275-0122 \\ moldovan@seas.smu.edu
}

\begin{abstract}
This paper presents the architecture, operation and results obtained with the LAsso Question Answering system developed in the Natural Language Processing Laboratory at SMU. To find answers, the system relies on a combination of syntactic and semantic techniques. The search for the answer is based on a novel form of indexing called paragraph indexing. A score of $55.5 \%$ for short answers and $64.5 \%$ for long answers was achieved at the TREC8 competition.
\end{abstract}

\section{Background}

Finding the answer to a question by returning a small fragment of a text, where the answer actually lies, is profoundly different from the task of information retrieval (IR) or information extraction (IE). Current IR systems allow us to locate full documents that might contain pertinent information, leaving it to the user to extract the answer from a ranked list of texts. In contrast, IE systems extract the information of interest, provided it has been presented in a predefined, target representation, known as template. The immediate solution of combining IR and IE techniques for question/answering (Q/A) is impractical since IE systems are known to be highly dependent on domain knowledge, and furthermore, the template generation is not performed automatically.

Our methodology of finding answers in large collections of documents relies on nat- ural language processing (NLP) techniques in novel ways. First, we perform the processing of the question by combining syntactic information, resulting from a shallow parse, with semantic information that characterizes the question (e.g. question type, question focus). Secondly, the search for the answer is based on a novel form of indexing, called paragraph indexing (Moldovan and Mihalcea 2000). Finally, in order to extract answers and to evaluate their correctness, we use a battery of abductive techniques (Hobbs et al.1993), some based on empirical methods, some on lexicosemantic information. The principles that have guided our paragraph indexing and the abductive inference of the answers are reported in (Harabagiu and Maiorano 1999).

\section{Overview of the LASSO Q/A System}

The architecture of LASSO (Moldovan, Harabagiu et. al 1999) comprises three modules: Question Processing module, Paragraph Indexing module and Answer Processing module. Given a question, of open-ended nature, expressed in natural language, we first process the question by creating a representation of the information requested. Thus we automatically find (a) the question type from the taxonomy of questions built into the system, (b) the expected answer type from the semantic analysis of the question, and most importantly, (c) the question focus defined as the main information required by that question. Furthermore, the Question Processing module also identifies the keywords from the question, which are passed to the Paragraph Indexing module, as illustrated by Figure 1. 


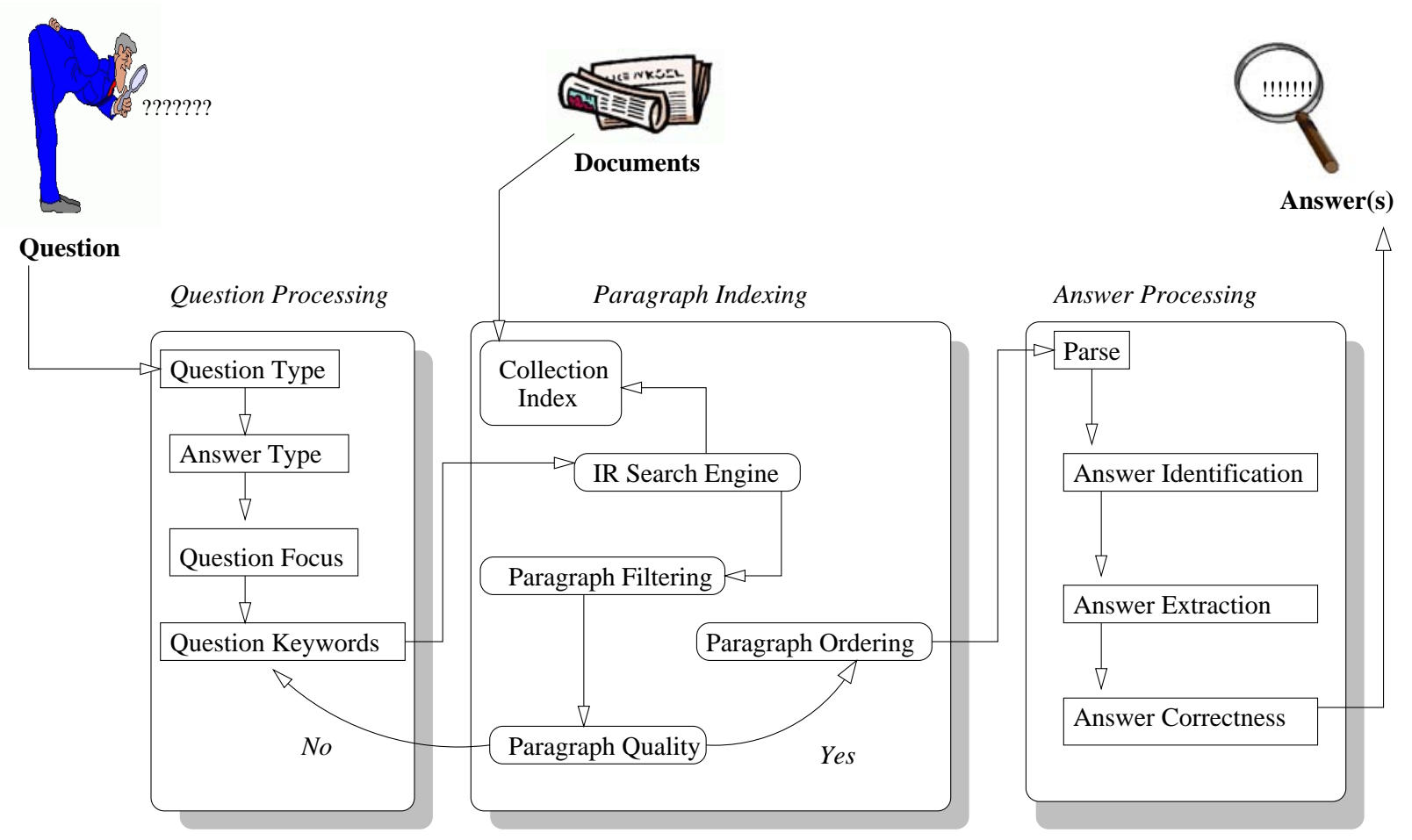

Figure 1: Architecture of the LAsso Q/A System

In LASSO, documents are indexed by a modified Zprise IR system available from NIST. Our search engine incorporates a set of Boolean operators (e.g. AND, OR, NOT, NEAR). We post-process the results of the IR search engine by filtering out the returns that do not contain all the keywords of a question in the same paragraph. This operation allows for on-the-fly generation of a paragraph index. The second important feature of the Paragraph Indexing module comes from the evaluation of the quality of the paragraphs. When the quality is satisfactory, the paragraphs are ordered according to a plausibility degree of containing the answer. Otherwise, we form new queries by adding or dropping keywords and resume the paragraph retrieval process. This loop generates a feed-back retrieval context that enables only a reasonable number of paragraphs to be passed to the $A n$ swer Processing module.

The advantage of processing paragraphs instead of full documents is a faster syntactic parsing. Our parses also involve Named
Entity recognitions and lexico-semantic resources useful in the answer extraction.

\section{Question Processing}

The role of the question processing module is to: (1) determine the question type, (2) determine the expected answer type, (3) build an answer focus, and (4) transform the question into queries for the search engine.

In order to find the right answer to a question from a large collection of texts, first we have to know what we should look for. The answer type can usually be determined from the question. For a better detection of the answer, the questions are first classified by their type: what, why, who, how, where questions, etc. A further classification follows to better identify the question type. Table 1 shows the classification for the 200 TREC- 8 questions.

We realized that the question type was not sufficient for finding answers. For the questions like Who was the first American in space?, the answer type is obvious: PERSON. However, this does not apply, for example, to 


\begin{tabular}{|c|c|c|c|c|c|c|}
\hline Q-class & Q-subclass & Nr.Q & $\begin{array}{l}\text { Nr. Q } \\
\text { answ. }\end{array}$ & Answer type & $\overline{\text { Example of question }}$ & $\overline{\overline{\text { Focus }}}$ \\
\hline \multirow[t]{5}{*}{ what } & & 64 & $5 \overline{54}$ & & & \\
\hline & basic what & 40 & 34 & $\begin{array}{l}\text { MONEY/NUMBER/ } \\
\text { DEFINITION/TITLE/ } \\
\text { NNP/UNDEFINED }\end{array}$ & $\begin{array}{l}\text { What was the monetary value of } \\
\text { the Nobel Peace Prize in 1989? }\end{array}$ & monetary value \\
\hline & what-who & 7 & 7 & $\begin{array}{l}\text { PERSON/ } \\
\text { ORGANIZATION }\end{array}$ & $\begin{array}{l}\text { What costume designer decided } \\
\text { that Michael Jackson should only } \\
\text { wear one glove? }\end{array}$ & costume designer \\
\hline & what-when & 3 & 2 & DATE & $\begin{array}{l}\text { In what year did Ireland elect } \\
\text { its first woman president? }\end{array}$ & year \\
\hline & what-where & 14 & 12 & LOCATION & What is the capital of Uruguay? & capital \\
\hline who & & $\overline{477}$ & $\overline{37}$ & $\begin{array}{l}\text { PERSON/ } \\
\text { ORGANIZATION }\end{array}$ & $\begin{array}{l}\text { Who is the author of the book } \\
\text { "The Iron Lady: A Biography } \\
\text { of Margaret Thatcher"? }\end{array}$ & author \\
\hline \multirow[t]{10}{*}{ how } & & $\overline{31}$ & $\overline{21}$ & & & \\
\hline & basic how & 1 & 0 & MANNER & How did Socrates die? & Socrates \\
\hline & how-many & 18 & 13 & NUMBER & $\begin{array}{l}\text { How many people died when } \\
\text { the Estonia sank in 1994? }\end{array}$ & people \\
\hline & how-long & 2 & 2 & TIME/DISTANCE & $\begin{array}{l}\text { How long does it take to travel } \\
\text { from Tokyo to Niigata? }\end{array}$ & - \\
\hline & how-much & 3 & 2 & MONEY/PRICE & $\begin{array}{l}\text { How much did Mercury spend } \\
\text { on advertising in 1993? }\end{array}$ & Mercury \\
\hline & $\begin{array}{l}\text { how-much- } \\
<\text { modifier }>\end{array}$ & 1 & 0 & UNDEFINED & $\begin{array}{l}\text { How much stronger is the new vitreous } \\
\text { carbon material invented by the Tokyo } \\
\text { Institute of Technology compared with } \\
\text { the material made from cellulose? }\end{array}$ & $\begin{array}{l}\text { new vitreous } \\
\text { carbon material }\end{array}$ \\
\hline & how-far & 1 & 1 & DISTANCE & How far is Yaroslavl from Moscow? & Yaroslav1 \\
\hline & how-tall & 3 & 3 & NUMBER & How tall is Mt. Everest? & Mt. Everest \\
\hline & how-rich & 1 & 0 & UNDEFINED & How rich is Bill Gates? & Bill Gates \\
\hline & how-large & 1 & 0 & NUMBER & $\begin{array}{l}\text { How large is the Arctic refuge to } \\
\text { preserve unique wildlife and wilderness } \\
\text { value on Alaska's north coast? }\end{array}$ & Arctic refuge \\
\hline where & & $\overline{22}$ & $\overline{16}$ & LOCATION & Where is Taj Mahal? & Taj Mahal \\
\hline when & & $\overline{19}$ & $\overline{13}$ & $\overline{\text { DATE }}$ & When did the Jurassic Period end? & Jurassic Period \\
\hline \multirow[t]{5}{*}{ which } & & $\overline{\overline{10}}$ & $\overline{8}$ & & & \\
\hline & which-who & 1 & 1 & PERSON & $\begin{array}{l}\text { Which former Klu Klux Klan } \\
\text { member won an elected office } \\
\text { in the U.S.? }\end{array}$ & $\begin{array}{l}\text { Klu Klux Klan } \\
\text { member }\end{array}$ \\
\hline & which-where & 4 & 3 & LOCATION & $\begin{array}{l}\text { Which city has the oldest relationship } \\
\text { as sister-city with Los Angeles? }\end{array}$ & city \\
\hline & which-when & 1 & 1 & DATE & $\begin{array}{l}\text { In which year was New Zealand } \\
\text { excluded from the ANZUS alliance? }\end{array}$ & year \\
\hline & which-what & 4 & 3 & $\begin{array}{l}\text { NNP/ } \\
\text { ORGANIZATION }\end{array}$ & $\begin{array}{l}\text { Which Japanese car maker had } \\
\text { its biggest percentage of sale in } \\
\text { the domestic market? }\end{array}$ & $\begin{array}{l}\text { Japanese } \\
\text { car maker }\end{array}$ \\
\hline \multirow[t]{4}{*}{$\overline{\text { name }}$} & & 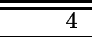 & $\overline{\overline{4}}$ & & & \\
\hline & name-who & 2 & 2 & $\begin{array}{l}\text { PERSON/ } \\
\text { ORGANIZATION }\end{array}$ & $\begin{array}{l}\text { Name the designer of the show } \\
\text { that spawned millions of plastic } \\
\text { imitations, known as "jellies"? }\end{array}$ & designer \\
\hline & name-where & 1 & 1 & LOCATION & $\begin{array}{l}\text { Name a country that is developing } \\
\text { a magnetic levitation railway system? }\end{array}$ & country \\
\hline & name-what & 1 & 1 & TITLE/NNP & $\begin{array}{l}\text { Name a film that has won } \\
\text { the Golden Bear in the Berlin } \\
\text { Film Festival? }\end{array}$ & film \\
\hline why & & $\overline{2}$ & 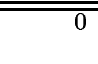 & REASON & $\begin{array}{l}\text { Why did David Koresh ask for a } \\
\text { word processor? }\end{array}$ & David Koresh \\
\hline$\overline{\text { whom }}$ & & $\overline{1.1}$ & $\overline{0}$ & $\begin{array}{l}\text { PERSON/ } \\
\text { ORGANIZATION }\end{array}$ & $\begin{array}{l}\text { Whom did the Chicago Bulls beat in } \\
\text { the } 1993 \text { championship? }\end{array}$ & Chicago Bulls \\
\hline & otal & $\overline{200}$ & $\begin{array}{r}153 \\
77 \% \\
\end{array}$ & & . & \\
\hline
\end{tabular}

Table 1: Types of questions and statistics. In this table we considered that a question was answered correctly if its answer was among top five ranked long answers. 
what questions, as what is ambiguous and it says nothing about the information asked by the question. The same applies to many other question types. The problem was solved by defining a concept named focus.

A focus is a word or a sequence of words which define the question and disambiguate the question by indicating what the question is looking for. For example, for the question What is the largest city in Germany?, the focus is largest city. Knowing the focus and the question type it becomes easier to determine the type of the answer sought, namely: the name of the largest city in Germany.

The focus is also important in determining the list of keywords for query formation. Often, many question words do not appear in the answer, and that is because their role is just to form the context of the question. For example, in the question In 1990, what day of the week did Christmas fall on?, the focus is day of the week, a concept that is unlikely to occur in the answer. In such situations, the focus should not be included in the list of keywords considered for detecting the answer.

The process of extracting keywords is based on a set of ordered heuristics. Each heuristic returns a set of keywords that are added in the same order to the question keywords. We have implemented eight different heuristics. Initially, only the keywords returned by the first six heuristics are considered. If further keywords are needed in the retrieval loop, keywords provided by the other two heuristics are added. When keywords define an exceedingly specific query, they are dropped in the reversed order in which they have been entered. The heuristics are:

- Keyword-Heuristic 1: Whenever quoted expressions are recognized in a question, all nonstop words of the quotation became keywords. - Keyword-Heuristic 2: All named entities, recognized as proper nouns, are selected as keywords.

- Keyword-Heuristic 3: All complex nominals and their adjectival modifiers are selected as keywords.

- Keyword-Heuristic 4: All other complex nominals are selected as keywords.

- Keyword-Heuristic 5: All nouns and their adjectival modifiers are selected as keywords.

- Keyword-Heuristic 6: All the other nouns recognized in the question are selected as keywords.

- Keyword-Heuristic 7: All verbs from the question are selected as keywords.

- Keyword-Heuristic 8: The question focus is added to the keywords .

Table 2 lists two questions from the TREC8 competition together with their associated keywords. The Table also illustrates the trace of keywords until the paragraphs containing the answer were found. For question 26, the paragraphs containing the answers could not be found before dropping many of the initial keywords. In contrast, the answer for question 13 was found when the verb rent was added to the Boolean query.

\begin{tabular}{||c|l||}
\hline \hline Q-26 & $\begin{array}{l}\text { What is the name of the "female" } \\
\text { counterpart to El Nino, which results in } \\
\text { cooling temperatures and } \\
\text { very dry weather? }\end{array}$ \\
\hline Keys & $\begin{array}{l}\text { female El Nino dry weather cooling temperatures } \\
\text { female El Nino dry weather cooling } \\
\text { female El Nino dry weather } \\
\text { female El Nino dry } \\
\text { female El Nino } \\
\text { female El }\end{array}$ \\
\hline \hline Q-13 & $\begin{array}{l}\text { How much could you rent a Volkswagen } \\
\text { bug for in 1966? }\end{array}$ \\
\hline Keys & $\begin{array}{l}\text { Volkswagen bug } \\
\text { Volkswagen bug rent }\end{array}$ \\
\hline \hline
\end{tabular}

Table 2: Examples of TREC-8 Question Keywords

\section{Paragraph Indexing}

\section{Search engine}

The Information Retrieval Engine for LASSO is related to the Zprise IR search engine available from NIST. There were several features of the Zprise IR engine which were not conducive to working within the design of LASSO. Because of this, a new IR engine was generated to support LASSO without the encumbrance of these features. The index creation was, however, kept in its entirety.

The Zprise IR engine was built using a cosine vector space model. This model does not allow for the extraction of those documents which include all of the keywords, but 
extracts documents according to the similarity measure between the document and the query as computed by the cosine of the angle between the vectors represented by the document and the query. This permits documents to be retrieved when only one of the keywords is present. Additionally, the keywords present in one retrieved document may not be present in another retrieved document.

LASSO's requirements are much more rigid. LASSO requires that documents be retrieved only when all of the keywords are present in the document. Thus, it became necessary to implement a more precise determinant for extraction. For the early work, it was determined that a Boolean discriminate would suffice provided that the operators AND and oR were implemented. It was also necessary to provide the ability to organize queries through the use of parentheses.

We opted for the Boolean indexing as opposed to vector indexing (Buckley et al.1998) because Boolean indexing increases the recall at the expense of precision. That works well for us since we control the retrieval precision with the PARAGRAPH operator which provides document filtering. In addition, the Boolean indexing requires less processing time than vector indexing, and this becomes important when the collection size increases.

To facilitate the identification of the document sources, the engine was required to put the document $i d$ in front of each line in the document.

The index creation includes the following steps: normalize the SGML tags, eliminate extraneous characters, identify the words within each document, stem the terms (words) using the Porter stemming algorithm, calculate the local (document) and global (collection) weights, build a comprehensive dictionary of the collection, and create the inverted index file.

\section{Paragraph filtering}

The number of documents that contain the keywords returned by the Search Engine may be large since only weak Boolean operators were used. A new, more restrictive operator was introduced: PARAGRAPH $n$. This op- erator searches like an AND operator for the words in the query with the constraint that the words belong only to some $n$ consecutive paragraphs, where $n$ is a controllable positive integer.

The parameter $n$ selects the number of paragraphs, thus controlling the size of the text retrieved from a document considered relevant. The rationale is that most likely the information requested is found in a few paragraphs rather than being dispersed over an entire document.

\section{Paragraph ordering}

Paragraph ordering is performed by a radix sort that involves three different scores: the largest Same_word_sequence-score, the largest Distance-score and the smallest Missing_keyword-score. The definition of these scores is based on the notion of paragraphwindow. Paragraph-windows are determined by the need to consider separately each match of the same keyword in the same paragraph. For example, if we have a set of keywords $\{k 1$, $k 2, k 3, k 4\}$ and in a paragraph $k 1$ and $k 2$ are matched each twice, whereas $k 3$ is matched only once, and $k_{4}$ is not matched, we are going to have four different windows, defined by the keywords: [k1-match1, k2-match1, k3], [k1-match2,k2-match1, k3], [k1-match1, k2match2, k3], and [k1-match2, k2-match2, k3]. A window comprises all the text between the lowest positioned keyword in the window and the highest position keyword in the window.

For each paragraph window we compute the following scores:

- Same_word_sequence-score: computes the number of words from the question that are recognized in the same sequence in the current paragraph-window.

- Distance-score: represents the number of words that separate the most distant keywords in the window.

- Missing_keywords-score: computes the number of unmatched keywords. This measure is identical for all windows from the same paragraph, but varies for windows from different paragraphs.

The radix sorting takes place across all the window scores for all paragraphs. 


\section{$5 \quad$ Answer Processing}

The Answer Processing module identifies and extracts the answer from the paragraphs that contain the question keywords. Crucial to the identification of the answer is the recognition of the answer type. Since almost always the answer type is not explicit in the question or the answer, we need to rely on lexicosemantic information provided by a parser to identify named entities (e.g. names of people or organizations, monetary units, dates and temporal/locative expressions, products and others). The recognition of the answer type, through the semantic tag returned by the parser, creates a candidate answer. The extraction of the answer and its evaluation are based on a set of heuristics.

\section{The Parser}

The parser combines information from broad coverage lexical dictionaries with semantic information that contributes to the identification of the named entities. Since part-ofspeech tagging is an intrinsic component of a parser, we have extended Brill's part-ofspeech tagger in two ways. First, we have acquired new tagging rules and secondly, we have unified the dictionaries of the tagger with semantic dictionaries derived from the Gazetteers and from WordNet (Miller 1995). In addition to the implementation of grammar rules, we have implemented heuristics capable of recognizing names of persons, organizations, locations, dates, currencies and products. Similar heuristics recognize named entities successfully in IE systems. Having these capabilities proved to be useful for locating the possible answers within a set of candidate paragraphs.

\section{Answer Extraction}

The parser enables the recognition of the $a n$ swer candidates in the paragraph. Each expression tagged by the parser with the answer type becomes one of the answer candidates for a paragraph. Similar to the paragraphwindows used in ordering the paragraphs, we establish an answer-window for each answer candidate. To evaluate the correctness of each answer candidate, a new evaluation metric is computed for each answer-window. We use the following scores:

- Same_word_sequence-score: it is computed in the same way as for paragraph-windows.

- Punctuation_sign-score: is a flag set when the answer candidate is immediately followed by a punctuation sign.

- Comma_3_words-score: measures the number of question words that follow the answer candidate when the latter is succeeded by a comma. A maximum of three words are sought.

- Same_parse_subtree-score: computes the number of question words found in the same parse sub-tree as the answer candidate.

- Same_sentence-score: computes the number of question words found in the same sentence as the answer candidate.

- Matched_keywords-score: computes the number of keywords matched in the answerwindow.

- Distance-score: adds the distances (measured in number of words) between the answer candidate and the other question words in the same window.

The overall score for a given answer candidate is computed by:

\begin{aligned} & \hline Combined-score $= 16 *$ Same_word_sequence-score + \\ &$+16 *$ Punctuation_sign-score + \\ &$+32 *$ Comma_3_words-score + \\ &$+16 *$ Same_parse_subtree-score + \\ &$+16 *$ Same_sentence-score + \\ &$+16 *$ Matched_keywords-score - \\ &$-4 * \sqrt{\text { Distance }- \text { score }} \\ &$\hline\end{aligned}

Currently the combined score represents an un-normalized measure of answer correctness. The answer extraction is performed by choosing the answer candidate with the highest score. Some of the scores approximate very simple abductions. For example, the recognition of keywords or other question words in an apposition determines the Punctuation_sign-score, the Same_parse_subtreescore, the Comma_3_words-score and the Same_sentence-score to go up. Moreover, the same sequence score gives higher plausibility to answer candidates that contain in their window sequences of question words that fol- 
low the same orders in the question. This score approximates the assumption that concepts are lexicalized in the same manner in the question and in the answer. However, the combined score allows for keywords and question words to be matched in the same order.

Table 3 illustrates some of the scores that were attributed to the candidate answers LASSO has extracted successfully. Currently we compute the same score for both short and long answers, as we analyze in the same way the answer windows.

\begin{tabular}{||l|l||}
\hline \hline Q.8 & $\begin{array}{l}\text { What is the name of the rare neurological } \\
\text { disease with symptoms such as : involuntary } \\
\text { movements (tics), swearing, and incoherent } \\
\text { vocalizations (grunts, shouts, etc)? }\end{array}$ \\
\hline \begin{tabular}{||l||} 
Answer \\
(short)
\end{tabular} & $\begin{array}{l}\text { Score: } 284.40 \text { who said she has both } \\
\text { Tourette's Syndrome and }\end{array}$ \\
\hline \hline Q.34 & $\begin{array}{l}\text { Where is the actress Marion Davies, } \\
\text { buried ? }\end{array}$ \\
\hline $\begin{array}{l}\text { Answer } \\
\text { (short) }\end{array}$ & $\begin{array}{l}\text { Score: 142.56 from the fountain inside } \\
\text { Hollywood Cemetery }\end{array}$ \\
\hline Q.73 & Where is the Taj Mahal? \\
\hline Answer & $\begin{array}{l}\text { Score: 408.00 list of more than 360 cities } \\
\text { throughout the world includes the Great } \\
\text { Reef in Australia, the Taj Mahal in India, } \\
\text { Chartre's Cathedral in France, and } \\
\text { Seregenti National Park in Tanzania. The } \\
\text { four sites Japan has listed include }\end{array}$ \\
\hline \hline Q.176 & $\begin{array}{l}\text { What is the nationality of Pope John } \\
\text { Paul II ? }\end{array}$ \\
\hline $\begin{array}{l}\text { Answer } \\
\text { (long) }\end{array}$ & $\begin{array}{l}\text { Score: 407.06 stabilize the country with its } \\
\text { help, the Catholic hierarchy stoutly held out } \\
\text { for pluralism, in large part at the urging of } \\
\text { Polish-born Pope John Paul II. When the } \\
\text { Pope emphatically defended the Solidarity } \\
\text { trade union during a 1987 tour of the }\end{array}$ \\
\hline \hline
\end{tabular}

Table 3: Examples of LASSO's correctness scores.

\section{Performance evaluation}

Several criteria and metrics may be used to measure the performance of a QA system. In TREC-8, the performance focus was on accuracy. Table 4 summarizes the scores provided by NIST for our system. The metric used by NIST for accuracy is described in (Voorhees and Tice 1999).

\begin{tabular}{||c|r|r||}
\cline { 2 - 3 } \multicolumn{1}{c|}{} & $\begin{array}{r}\text { Percentage of } \\
\text { questions in top 5 }\end{array}$ & NIST score \\
\hline \hline Short answer & $68.1 \%$ & $55.5 \%$ \\
\hline Long answer & $77.7 \%$ & $64.5 \%$ \\
\hline \hline
\end{tabular}

Table 4: Accuracy performance

Another important performance parameter is the processing time to answer a question.

\begin{tabular}{||l|r||}
\hline Type of processing & Time percentage \\
\hline \hline Question processing & $0.1 \%$ \\
\hline Paragraph search & $34.0 \%$ \\
\hline Paragraph ordering & $6.0 \%$ \\
\hline Answer extraction & $59.9 \%$ \\
\hline \hline
\end{tabular}

Table 5: Time performance

On the average, the processing time per question is 61 sec., and the time ranges from 1 sec. to 540 sec. There are four main components of the overall time: (1) question processing time, (2) paragraph search time, (3) paragraph ordering time, and (4) answer extraction time. Table 5 summarizes the relative time spent on each processing component. The answer extraction dominates the processing time while the question processing part is negligible.

\section{Conclusions}

In principle, the problem of finding one or more answers to a question from a very large set of documents can be addressed by creating a context for the question and a knowledge representation of each document and then match the question context against each document representation. This approach is not practical yet since it involves advanced techniques in knowledge representation of open text, reasoning, natural language processing, and indexing that currently are beyond the technology state of the art. On the other hand, traditional information retrieval and extraction techniques alone can not be used for question answering due to the need to pinpoint exactly an answer in large collections of open domain texts. Thus, a mixture of natural language processing and information retrieval methods may be the solution for now.

In order to better understand the nature of the QA task and put this into perspective, we offer in Table 6 a taxonomy of question answering systems. It is not sufficient to classify only the types of questions alone, since for the same question the answer may be easier or more difficult to extract depending on how the answer is phrased in the text. Thus we classify the QA systems, not the questions. We provide a taxonomy based on three crite- 


\begin{tabular}{|c|c|c|c|c|}
\hline Class & $\overline{\mathbf{K B}}$ & Reasoning & NLP/Indexing & Examples and Comments \\
\hline 1 & dictionaries & $\begin{array}{l}\text { simple } \\
\text { heuristics, } \\
\text { pattern } \\
\text { matching }\end{array}$ & $\begin{array}{l}\text { complex noun, } \\
\text { apposition, } \\
\text { simple } \\
\text { semantics, } \\
\text { keyword } \\
\text { indexing }\end{array}$ & $\begin{array}{l}\text { Q33: What is the largest city in Germany? } \\
\text { A: .. Berlin, the largest city in Germany.. } \\
\text { Answer is: simple datum or list of items found verbatim in } \\
\text { a sentence or paragraph. }\end{array}$ \\
\hline 2 & ontologies & $\begin{array}{l}\text { low } \\
\text { level }\end{array}$ & $\begin{array}{l}\text { verb } \\
\text { nominalization, } \\
\text { semantics, } \\
\text { coherence, } \\
\text { discourse }\end{array}$ & $\begin{array}{l}\text { Q198: How did Socrates die? } \\
\text { A: .. Socrates poisoned himself.. } \\
\text { Answer is contained in multiple sentences, scattered throughout } \\
\text { a document. }\end{array}$ \\
\hline 3 & $\begin{array}{l}\text { very large } \\
\mathrm{KB}\end{array}$ & $\begin{array}{l}\text { medium } \\
\text { level }\end{array}$ & $\begin{array}{l}\text { advanced nlp, } \\
\text { semantic } \\
\text { indexing }\end{array}$ & $\begin{array}{l}\text { Q: What are the arguments for and against prayer in school? } \\
\text { Answer across several texts. }\end{array}$ \\
\hline 4 & $\begin{array}{l}\text { Domain KA } \\
\text { and } \\
\text { Classification, } \\
\text { HPKB }\end{array}$ & $\begin{array}{l}\text { high } \\
\text { level }\end{array}$ & & $\begin{array}{l}\text { Q: Should Fed raise interest rates at their next meeting? } \\
\text { Answer across large number of documents, domain specific } \\
\text { knowledge acquired automatically. }\end{array}$ \\
\hline 5 & $\begin{array}{l}\text { World } \\
\text { Knowledge }\end{array}$ & $\begin{array}{l}\text { very high } \\
\text { level, } \\
\text { special } \\
\text { purpose }\end{array}$ & & $\begin{array}{l}\text { Q: What should be the US foreign policy in the Balkans now? } \\
\text { Answer is a solution to a complex, possible developing scenario. }\end{array}$ \\
\hline
\end{tabular}

Table 6: A taxonomy of Question Answering Systems. The degree of complexity increases from Class 1 to Class 5, and it is assumed that the features of a lower class are also available at a higher class.

ria that we consider important for building question answering systems: (1) knowledge base, (2) reasoning, and (3) natural language processing and indexing techniques. Knowledge bases and reasoning provide the medium for building question contexts and matching them against text documents. Indexing identifies the text passages where answers may lie, and natural language processing provides a framework for answer extraction.

Out of the 153 questions that our system has answered, 136 belong to Class 1 , and 17 to Class 2. Obviously, the questions in Class 2 are more difficult as they require more powerful natural language and reasoning techniques.

As we look for the future, in order to address questions of higher classes we need to handle real-time knowledge acquisition and classification from different domains, coreference, metonymy, special-purpose reasoning, semantic indexing and other advanced techniques.

\section{References}

Chris Buckley, Mandar Mitra, Janet Walz and Claire Cardie. SMART Hight Precision: TREC
7. In the Proceedings of the Text Retrieval Conference TREC- $7,1998$.

Sanda Harabagiu and Steven Maiorano. Finding answers in large collections of texts: paragraph indexing + abductive inference. Working Notes of the Fall AAAI Symposium on Question Answering, November 1999.

Jerry Hobbs, Mark Stickel, Doug Appelt, and Paul Martin. Interpretation as abduction. Artificial Intelligence, 63, pages 69-142, 1993.

G.A. Miller. WordNet: A Lexical Database. Communication of the ACM, vol 38: No11, pages 39-41, November 1995.

Dan Moldovan, Sanda Harabagiu, Marius Pasca, Rada Mihalcea, Roxana Girju, Richard Goodrum and Vasile Rus LASSO: A Tool for Surfing the Answer Net In Proceedings of TREC-8, pages 65-74, 1999. http://trec.nist.gov/pubs/trec8/papers/smu.ps

Dan Moldovan and Rada Mihalcea. Improving the search on the Internet by using WordNet and lexical operators In IEEE Internet Computing, vol.4, no.1, pages 34-43, 2000.

Ellen M. Voorhees and Dawn M. Tice, The TREC-8 Question Answering Track Evaluation In Proceedings of TREC-8, pages 41-64, 1999. http://trec.nist.gov/pubs/trec8/papers/qa8.ps 